\title{
COORDINATING EMERGENCY RESPONSE BY COMPETENT TEAMS
}

\author{
Danilo Hasse ${ }^{1}$, Fernando A. O. Gauthier ${ }^{1}$, Carlos Roberto de Rolt ${ }^{2}$ \\ and Gisiela Hasse Klein ${ }^{2}$ \\ ${ }^{l}$ Departamento de Engenharia e Gestão do Conhecimento, Universidade Federal de Santa Catarina - \\ UFSC Campus Universitário Reitor João David Ferreira Lima - Florianópolis - Santa Catarina, Brasil \\ ${ }^{2}$ Centro de Ciências da Administração e Socioeconômicas, Universidade do Estado de Santa Catarina- \\ UDESC Campus Av. Madre Benvenuta, 2037 - Florianópolis - Santa Catarina, Brasil
}

\begin{abstract}
ICTs and social networks can contribute to the emergence of creating competent teams for emergency response. For example, during disasters such as Pakistan flood of 2010, Japan tsunami of 2011 and Thailand flood of 2011, On-Line Social Networks (OSNs) have served as a main technology for numerous people seeking to share information about their personal status, to request resources, or to report the status of their community. They can be used at least for three main functions: detecting emergencies, disseminating information, and managing emergencies. On the other hand, the combination of Internet and mobile technology generated smart devices with associated sensor technologies that are becoming crucial parts in delivering supports during disaster and emergency situation. However, such use of mobile devices usually requires a reliable support system from crowdsensing technologies and back-end intelligent systems. In this way, the mobile crowdsensing must be designed with focus on mechanisms to identify and localize survivors and first responders in an incident zone, as well as mechanisms for competence characterization provided by social networks to facilitate coordination for individuals as well as crowds. These main technologies, the crowdsensing and social networks combined with semantic web and ontologies can provide a complete emergency response system that is the purpose of the CO-SEMIWA platform. This platform permits an interaction among participants to create dynamically competent teams where all participants perform tasks and solve problems in a specific emergency context and situation.
\end{abstract}

\section{KEYWORDS}

Emergency Response, Social Networks, Crowdsensing; Ontologies, Expertise Location, Competent Teams 


\section{INTRODUCTION}

During disasters such as Pakistan flood of 2010, Japan tsunami of 2011 and Thailand flood of 2011, On-Line Social Networks (OSNs) have served as a go-to-technology for numerous people seeking to share information about their personal status, to request resources, or to report the status of their community. In part, this is due to OSNs in providing an innovative platform that enables users to easily share information without the constraints imposed by distance, time, or space. Also, most platforms (e.g., Facebook, Twitter, LinkedIn etc.) are designed to work across devices (e.g., mobile devices, computers etc.).

Consequently, government, not-for-profit organizations, and citizens have found innovative ways to leverage OSNs tools to share information and coordinate action during and after disasters. OSNs can be used in at least three distinct ways to manage emergencies. They can be used while detecting emergencies, disseminating information, and managing emergencies (Carter et al. 2014).

ICTs and Social Networks can contribute to the emergence of creating competent teams for Emergency Response using emergent technologies such as mobile crowdsensing, Wearable Sensors Devices (WSD) and semantic web / ontologies. These technologies enable to provide a complete system and infrastructure to manage emergency situations in the response phase of the emergency management. Thus, with notion of competence the interaction among participants can create dynamically teams from a previously known emergency response platform where all participants perform tasks and solve problems in a specific emergency context and situation.

This paper presents a description of a framework for extraction competence profiles by social networks and creation of competent teams in the scope of CO-SEMIWA's project a semantic middleware and mobile crowdsensing platform for emergency response. For this purpose, a literature review was carried out on topics related to the goals of this proposed system and are grouped into two main sections: section 2 presents the Emergency Management and Response; and section 3, describes issues for Creating Competent Teams. The section 4 details our CO-SEMIWA's Framework to deal with team formation over a social network such as LinkedIn and the section 5, the Conclusion.

\section{EMERGENCY MANAGEMENT AND RESPONSE}

Every year, millions of people are affected by natural disasters such as earthquakes, tsunamis, volcano eruptions, hurricanes, tornados and floods, and governments all around the world spend huge amounts of resources on the reconstruction and the preparation for such calamities. In 2013 alone, 97 million victims were affected by 330 natural disasters and the economic damage amounted to 156.6 billion dollars (Rosas et al. 2016).

In 1999, The United Nation's International Strategy for Disaster Reduction (UNISDR) was established to coordinate efforts on disasters risk initiatives around the world. In 2009, the UNISDR published the UNISDR Terminology on Disaster Risk Reduction that aims to promote common understanding and common usage of disaster risk reduction concepts and to assist the disaster risk reduction efforts of authorities, practitioners and the public. According to UNISDR (2009) this document is still valid for reference, where the term Emergency Management was defined as: "the organization and management of resources and 
responsibilities for addressing all aspects of emergencies, in particular preparedness, response and initial recovery steps".

More recently, the third United Nations World Conference on Disasters Risk Reduction (WCDRR) was held in Sendai, Japan, in 2015 and the Sendai Framework for Disasters Risk Reduction (DRR) 2015-2030 was announced as a successor instrument to the Hyogo Framework for Action (HFA). The UNISDR is the key acting body in the United Nations and serve a focal point for coordination of disaster risk reduction as well has been tasked to support the implementation and review of de Sendai Framework.

Therefore, an emergency is a situation that poses an immediate risk to human health, life, and property, which requires urgent interventions to prevent its worsening.

In general, four predominant phases characterize the Emergency Management and Response action, presented by several works in the literature (Vivacqua and Borges, 2001; Moore, 2008; Araujo and Borges, 2012; Pavković and Milenković, 2014; Cova, 1999; Harb and Dell'acqua, 2017; Nazer, 2017; Li et al, 2017) as a cycle containing: mitigation, preparedness, response and recovery. Briefly, the Mitigation phase provides redundancy in preparedness plans to avoid re-occurrence of a disaster or to diminish the destructive impact. Preparedness phase details on exact command and control actions to be performed by responsible Emergency Management Agencies (EMAs) and first responders (EFRs) in response to a disastrous situation. Response phase puts in practice the plans conceived during the preparedness phase in order to restore order, help the affected people, and neutralize the most immediate hazards (life, health, property, and environment). The recovery phase after the initial relief has been provided and the goal is returning life to normal or improved circumstances.

Each phase presents a specific characteristic of activities, requirements of resources and demands of systems. For instance, the response phase immediately after the event includes multiple functions, such as damage assessment, response-needs assessment, response prioritization, coordination and mobilization of rescue operations, implementation of resource, logistic and evacuation planning, situation monitoring, and timely information dissemination to citizens and organizations (Mehrotra et al, 2013). The complexity of actions taken during emergencies depends upon the types of disasters, the severity of the crisis and the entities involved. Depending upon the disaster's magnitude and scope, response might involve multiple levels of the government (city, county, state, and federal agencies), national and international partners, public authorities, commercial entities, hospitals and health organizations, volunteer organizations, media organizations, and the public.

\subsection{Response Phase}

The Response phase is arguably the most complex and, therefore, the most studied phase. Some of its characteristics are: unpredictability, speed of events, number of people involved, short decision and action times, unavailability of resources, uncertainty about the situation, pressure and stress on those involved (Vivacqua and Borges, 2001).

This phase aims at saving lives and preventing major economic losses and environmental problems. It begins immediately after an event with adverse impacts on a community happens, and ends when the situation has been stabilized. It consists, in principle, on putting into action plans that had been previously developed and exercised (in the preparation phase) (Vivacqua and Borges, 2001). 
IADIS International Journal on Computer Science and Information Systems

The so-called Emergency Response Systems (ERSs) resulted from the need for a new method to manage rapidly moving wildfires in the early 1970s. At that time, emergency managers faced a number of problems, such as too many people reporting to one supervisor, lack of reliable and accurate incident information, and inadequate and incompatible communications (Liu et al, 2015). This process is itself a cycle composed of some steps: the situation is understood and decisions are made; the selected actions are performed, which affect, along with external events, the situational context; this generates a need to update information on the emergency so that new decisions can be made (Vivacqua and Borges, 2001).

Therefore, immediate knowledge of the amount and the level of damage present of particular interest to both humanitarian and financial aid efforts. However, usually the necessary damage-related information is difficult to attain from ground sources, as in situ data collection faces challenges including time constraints, area accessibility, and the physical safety of surveyors. The rapid and efficient information for response activities after a disaster have a major impact on the number of fatalities. Remote sensing provided by mobile applications (e.g. crowdsensing) can play a guiding role for Gathering data in such emergency situations by offering real-time synoptic views on the affected areas (Harb and Dell'acqua, 2017). Real-time data collection and validation involves techniques to gather heterogeneous data from sources of qualitatively different kinds. The proposed techniques address three barriers to real-time data access: i) degradation at the site of the incident or in transmission; ii) uncertainty due to stress or disinformation; and, iii) varying levels of significance based on context and data veracity. Provisioning techniques will dynamically configure communication capacity between the site(s) of an incident and locations where analytics will be done; and to dynamically provision the storage and computational resources needed to carry out the analytics. Analytics techniques will focus on learning the type, scope, severity and dynamic properties of incidents from heterogeneous data (Abu-Elkheir et al, 2016).

However, as each emergency is unique, plans usually cannot be executed directly and require adaptation. Therefore, personnel involved also need to take actions and create new procedures during the response to the emergency (Vivacqua and Borges, 2001). The effectiveness of response depends on the ability to quickly assess the situation and mobilize the necessary resources (Weaver et al, 2016). That's why in general detecting and mapping a hazard is one important step in response. Diversities of disasters and emergencies call for distinct detection and identification processes ( $\mathrm{Gu}$ et al, 2017). With modern technology, intelligent detection devices and networking, aware responders and emergency managers first become aware of what just happened. Recent advances in mobile communication coupled with increased computational capabilities of mobile devices present an unprecedented opportunity for situation-aware and mobile applications. These applications can be very beneficial to emergency medical services as they are able to provide emergency teams with ready access to the status and location of dispersed emergency resources using their mobile phones. This is particularly important because medical response is usually tiered with an initial urgent response and assessment team relying on subsequent specialist teams if required. One of the most recent and potential approaches for developing distributed and mobile applications that enable information sharing and collaboration is service-oriented computing (Haghighi et al, 2010).

During response, the emergency team is dealing with the situation as it evolves. It should be noted that actions reflect on the situation, so the context is constantly changing, not only due to environmental (uncontrollable) events, but also due to actions in response to the 
situation, that attempt to bring it under control. It is important to keep up with the evolving emergency situation, in order to take appropriate action, but it is hard for a team to keep the environment under watch at all times (Vivacqua and Borges, 2001).

For instance, the response phase immediately after the event includes multiple functions, such as damage assessment, response-needs assessment, response prioritization, coordination and mobilization of rescue operations, resource and logistic planning, evacuation planning, situation monitoring, and timely information dissemination to citizens and organizations (Cong et al. 2015).

\subsection{Coordinating Emergency Assistance}

We concentrate in this paper on the coordinating emergency assistance efforts for the response phase of the emergency management cycle. In this issue the interventions are organized as a process that is usually described in an emergency control plan, named an emergency response process. In this process, all individuals, groups, and communities manage hazards in an effort to avoid or reduce the impact of disasters. It is based on the idea that an emergency response process is quite similar to a business process and, therefore, can be modeled as a workflow (Cong et al. 2015).

Therefore, the reliability, relevance and timeliness of data are crucial because they will foster effective coordination between the national and local governments, international organizations and non-governmental actors, and in particular with affected households and communities. Technology is believed to speed up the coordination efforts to relief the emergency situation and rescue victims for saving lives. The combination of internet and mobile technology generated smart devices with associated sensor technologies that are becoming crucial parts in delivering supports during the disaster and emergency situation. However, such use of mobile devices usually requires a reliable support system from crowdsensing technologies and back-end intelligent systems (Geumpana et al. 2015).

In this way, a mobile crowdsensing system is crucial for coordinating emergency efforts by a crowd management component with focus on mechanisms to identify and localize survivors and first responders in an incident zone, as well as mechanisms for competence characterization by social networks to facilitate coordination for individuals as well as crowds. Crowdsourced data from sources verified to be in the impact zone of an incident can be collected and integrated to the scene of the incident. On the other hand, several works relate the social networks as one the most promising applications in the emergency management and response (Geumpana et al. 2015). Finally, the semantic web technology and ontologies have been used in the emergency management and response area. Ontologies can also unify and normalize data from different resources, e.g., social networks, syntactically and semantically and associate it with emergency domain knowledge. They help to create meaningful relationships between information resources and to allow machines to process, infer, or combine the information from different sources automatically into a consistent body of knowledge (Moi et al. 2016). In this way, several works in the literature can be founded using Semantic Web and Ontologies for emergency management systems (Moi et al. 2016).

Thereby, the mobile crowdsensing and social networks combined with semantic web and ontologies are the main components in the CO-SEMIWA platform and this integration represents a novel approach for emergency response in the literature. This paper proposes a semantic middleware framework for the back-end intelligence of the CO-SEMIWA platform providing the possibility to enhance the creation of competent teams for emergency response as described in the next sections. 


\section{CREATING COMPETENT TEAMS}

In this section, we reviewed several works to creating competent teams in the context of social networks and emergency management and response systems. We have concentrated on the most prominent works describing the main issues related to this effort in the next topics, as follows: 3.1 Team Formation with Social Networks; and, 3.2 Tasks Assignment and Delegation.

Creating competent teams in a disaster and emergency scenario involves many types of stakeholders, as well as numerous people and organizations within each stakeholder category: victims, first-responders, Emergency Management Agencies - EMAs, volunteers, etc. Dozens of municipal, county, state, and federal agencies have formal response responsibilities. There are also informal responders such as NGOs (for example, the Red Cross), local and regional volunteer relief groups, social media networks (for example, Facebook and Twitter), and individual citizens. Industries such as public utilities, construction, and insurance also play a major role (Murphy, 2016).

Emergency management involves multiple stakeholders working together before, during and/or after an emergency with the objective to minimize the negative impacts and recover as fast as possible. Three main groups of stakeholders are (Luna and Pennock, 2015): i) Government agencies, ii) Private volunteering agencies and iii) Civilians. Communication and coordination among these groups is crucial for emergency management success. Proper coordination must exist during an emergency crisis in order to implement an effective response. Governments, private organizations and communities collaborate to minimize the impact of a disaster. However, it has been recognized that during an emergency, organizations do not react in the most efficient manner. Interoperability issues include misinformation, rumors, and cascading information flow.

The integration of this diversity of stakeholders demands a type of systems that have capabilities to establish very widespread communications, interoperability, data integration and usability interfaces. Here is a real challenge involving different information patterns to communicate and manage disaster process requiring decision making under extreme conditions.

Social challenges also emerge when may intervene residents' daily life or affect public behavior. Actually, there are debates on the ethical issues on adopting such systems. Another issue is whether emergency warning should guide the public. Sometimes residents might refused to evacuate. To the opposite, the local government may order public evacuation although the legal authority may be lacking (Moore, 2008).

Disaster and emergency response networks are characterized by heterogeneity in their network relationships (for example, direction and control versus voluntary coordination, or formal or contractual versus informal relationships) and a shifting composition as new organizational entities join the network in response to changing conditions and disaster related demand (Jaimes et al., 2015).

Organizational challenges arise from several reasons. One problem is the connections among organizations in terms of responsibilities. For example, the probability of recognizing a future disaster is determined by to what degree the indicator of the potential threat can be detected. So, different organizations may detect different phenomenon. Another problem is in disaster response, we need to make decision based on after, dynamic, ad-hoc organization. As we use collaborative technologies such as mobile applications, Web-based systems, and 
communications networks such as wireless mesh networks, there may be too many resources and too much information that restricts our ability to manage emergency and aspects the capacity of emergency communication systems $(\mathrm{Li}, 2011)$.

Roles played by citizens action in disaster and emergency are widely documented in disaster research. The researches questioning the popular perception that disasters unleash chaos and disorganization, with citizens becoming passive victims, panic-stricken, or engaging in anti-social behaviour such as looting (Whittaker et al, 2015).

Rather, individuals and groups have generally been found to become more cohesive than in "normal" times, commonly working together to overcome disaster. Emergent groups often form during or immediately after the emergency period, before established and extending organizations arrive. These groups often play critical "first responder" roles such as initial search and rescue, providing first aid to victims, and assessing damages and community needs. Like extending organizations, they can pose significant challenges for emergency managers. Emergent volunteerism involves new forms of volunteering that occur in response to unmet needs, whether perceived or real. Researchers and emergency managers shave tended to focus on challenges associated with "spontaneous" volunteers, usually once an emergency or disaster has begun (Whittaker et al, 2015).

The Responders must be able to comprehend, visualize, and trust the data they collect. They will experience physiological and cognitive deficits due to the physical and emotional demands of working around the clock in a tragic situation. Computational tools that support decision makers are thus essential (Murphy, 2016).

Users Response personnel might need to share information across diverse, loosely coupled, emergent multi-organizational networks that lack centralized control, in which different entities play different roles in response activities, have different needs and urgencies, different cultures, and potentially vastly different capabilities with respect to technology use (Jaimes, 2015).

\subsection{Team Formation with Social Networks}

The social network of an individual plays a perfect role as a medium for the spread of information, ideas and influence among the team members. Awal and Bharadwaj (2014) present a comprehensive literature review of this area and propose a model for team formation.

Lappas et al. (2009) introduced the problem of team formation in the context of social networks, and thereafter, much work has been recently carried out in this area by considering various algorithms with different objectives (e.g., minimizing the coordination costs, covering all skills, etc.). The problem can be stated as: "given a particular task and a set of experts, the problem is to identify the right team of experts that can collectively perform the given task in an effective manner by covering all the required skills". Li and Shan (2010) extended the work further by considering the problem of generalized tasks (the type of task where a designated number of experts is needed for each required skill), identifying a relevant set of experts for a particular task requires the discovery of an optimal configuration of a team. Wi et al. (2009) consider the two elements of the competence of a team in a social network setting, that is: i) knowledge competence: personal knowledge and knowledge from the social network; ii) collaboration competence: based on social network analysis measures (density, degree centrality and closeness centrality). Bonchi et al. (2011) show that Social Network Analysis 
IADIS International Journal on Computer Science and Information Systems

(SNA) help in visualizing and understanding the roles and relationships that facilitate or hinder the collaboration and sharing of information and knowledge in an organization.

The Team Formation algorithms also have the first work with Lappas et al. (2009) in the team formation problem in the presence of a social network and presents greedy algorithms for minimizing the diameter and the cost of the minimum spanning tree (MST) induced by the team. Lappas et al. (2009) impose the strong assumption that a skill requirement of a task can be fulfilled by a single person. On the other hand, the solutions obtained by all these algorithms (including the MST algorithm) can be shown to be connected subgraphs if the underlying social graph is connected. Gajewar and Sarma (2012) explore the usefulness of the density based objective in finding strongly connected teams. Anagnostopoulos et al. (2012) considered an online team formation problem where tasks arrive in a sequential manner and teams have to be formed minimizing the (maximum) load on any expert across the tasks.

\subsection{Tasks Assignment and Delegation}

Assignment of tasks to people, following different kind of strategies and patterns has already been studied extensively in the literature. For example, Palen and Liu (2007) describe the emergence of information pathways for public participation in emergency response, and discuss the need to coordinate improvised activities between temporary organizations and formal response teams. They include examples of how the majority of victims in disaster are saved by local, ad hoc volunteer groups.

In general, these approaches can divided into three categories (Bessai and Charoy 2016): i) the mono-criterion approaches where the objective is to minimize only one criterion (generally the overall execution time); ii) the multi-criteria approaches where several objectives often conflicting are considered simultaneously; and iii) the constraint where the initial multi-objectives problem is transformed to the mono-criterion one (only one objective is considered and the rest of objective functions are transformed to constraints). These approaches have been proved to be effective in the case where all tasks are automated. A solution that is widely used to cope with this issue is to call for more resources to deal with the new load and to delegate tasks to these resources. These types of resources can be found using crowdsourcing platforms in an elastic way. However the problem with crowdsourcing is to find resources with the right skills and to trust the quality of output by unknown people. For instance, in case of a humanitarian crisis, it is usual to call to social networks and the crowd to execute task instead of responders (Bessai and Charoy 2016).

In this sense, the Dynamic Task Allocation is other important issue to the Task Assignment. Task monitoring and reassignment in large-scale disaster response, in rapidly changing environments, in the presence of data explosion is challenging, since many events may get lost in such volume of data. Intelligent decisions need to be made quickly, evaluated and changed depending on updated information. In emergency response, where the user's environment changes rapidly, mobility context is an essential element that needs to be part of the decision making process. However, there is a lack of systems that continuously monitor the mobility context of task owners to determine the quality or progress of the task, and whether additional resources should be added, removed, or reassigned to someone else who could do it faster and within the given time constraints (Luqman and Griss 2010).

Government's studies show that existing communication systems used for emergency response are ineffective and incompatible across teams. Recently, the interoperability of 
existing emergency response communications has been highlighted. Furthermore, the closed communication systems used by first responders and emergency response personnel are often incompatible with those used by volunteers. Current technologies are also inadequate for spontaneously formed disaster response teams. Well informed decisions must be made rapidly and reevaluated periodically to gauge their effectiveness (Luqman and Griss 2010).

Achieving automation in the task delegation process can be done by using a number of methods, most of them quite new and derived from the field of artificial intelligence. However, the chosen model was the usage of classic iterative algorithms that create automation with respect to task delegation by applying a set of instructions to properly stored, sorted and indexed data available on the project's back-end database. In general, project data is being distributed across multiple nodes via NoSQL databases where for this purpose are better suited than classic relational databases (Pop and Boian 2014).

\section{CO-SEMIWA: A SEMANTIC MIDDLEWARE FOR EMERGENCY RESPONSE}

The COLLEGA Semantic Middleware (CO-SEMIWA) is a semantic middleware for collaborative assistance in emergency response scenarios. The system is context-aware collecting emergency information from a crowdsensing platform and Wearable Sensors Devices (WSD) connected in the monitored people, extracting competence information from social networks such as LinkedIn to know potential participants by profiles and providing semantic information to build automatically teams for emergency response.

The CO-SEMIWA Architecture can be visualized in Figure 1, where its functional layers and modules are shown. In our research work, the architecture model is layered into two layers: the COLLEGA Middleware - the Application Layer (AL) and COLLEGA Semantic Middleware - the Semantic Layer (SL).

The COLLEGA middleware (AL) is our previous crowdsensing platform (De Rolt et al. 2017) to support mobile participatory healthcare communities and a reference middleware architecture, called by acronym COLLEGA (COLLaborative Emergency Group Assistant), for handling anytime and anywhere emergency situations using a crowdsensing to manage the overall life cycle of an emergency situation, from alarm detection and understanding to the formation of virtual mobile communities formed by passing-by users.

Initially, the social network platform is composed of potential participants who do not know each other, and do not share a common interest in the virtual or physical world. Participants are physically in the same place at that time, they have no degree of previous cooperation and don't have any kind of communication synchronously or asynchronously with the WSD user but are users of mobile computing systems, such as smartphones and choose to install a crowdsensing APP (COLLEGA) of emergency response public service.

The Figure 1 also represents the user that consciously opted to use a remote monitoring system - WSD and, in doing so, joined a pre-existing virtual community with known and registered members. Participants of this pre-existing virtual community opted for some reason (family, friends, volunteers or EMAs) to join the community. Thus, the Application Layer $(\mathrm{AL})$ is the core of COLLEGA's functionalities to form the overall structure with modules that manage the overall cycle of an emergency. The AL Layer encompasses multiple devices like 
IADIS International Journal on Computer Science and Information Systems

smartphones. The "Applications" on the AL Layer are responsible for providing visualizations of ongoing processes or assist the users in sending control instructions.

The application description is an instance of our provided application model and holds meta-information about the executing device, the associated processes and a unified identifier that finds corresponding distributed applications on several devices. The Application Layer executes functions and provides services for the system.

The modules and respectively functionalities of the Application Layer are: a) Monitoring System (MOS): getting and analyzing data collected from sensors and receiving and sending emergency alerts by individuals; b) Emergency Context Analyzer (ECA): detecting and classifying emergency situations and choosing the most appropriate control plan; c) Potential Participant Analyzer (PCA): automatically identifying potential participants; d) Virtual Community Manager (VCM): ensuring that potential participants can properly access monitored emergency data and monitoring task implementation; and, e) SFW: providing information about the monitored emergency in a secure way to participants.

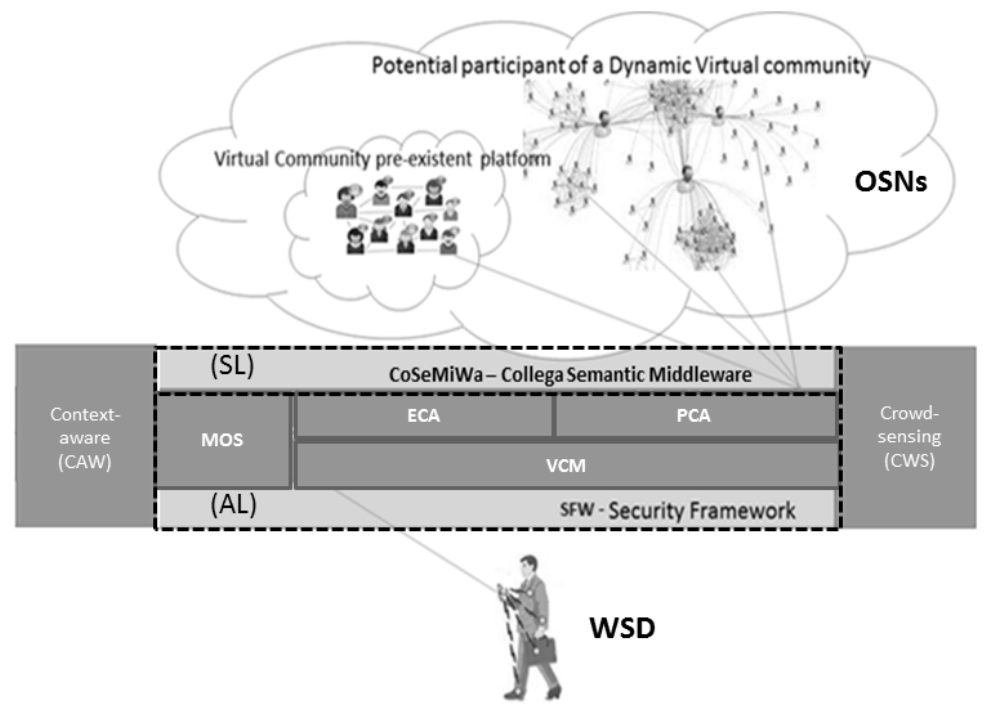

Figure 1. CO-SEMIWA architecture

The COLLEGA crowdsensing platform (CWS) interacts with the Application Layer through execution of functions to collect data of all available users in the emergency area. From technological point of view, a complete crowdsensing platform consists of an app running on smartphones and a web application on the back-end. Main support functions include: management of the crowdsensing requests (called tasks); sensing of data collected either passively (automatically via smartphone sensors), actively (with direct user participation), or with a mix of these modes; and evaluating assignment of tasks to users for future crowdsensing activities. The crowdsensing client is the component that takes care of receiving tasks, asking users whether they want to run them, managing data collection, and uploading results. Functionally, a crowdsensing client comprises two main components: the task management component and the sensing management component. These components are 
responsible for both interacting with users and accessing smartphone sensors. The server side provides management, storage, and analysis of crowdsensed data. At the highest level comprises two main parts: the back-end and the crowdsensing manager (Corradi et al. 2015).

As explained above the WSD and CWS platforms connect sensors through mobile phones with relevant data about emergencies to be managed by COLLEGA application layer (AL). However, the selectively of OSN profiles is an important feature for creating competent teams to execute the control plans.

In this sense, our previous work (Hasse et al. 2017) studied relevant references of competence developing a new proposed approach for the competence in the professional social networks contributing with a model of "Competence Networks" with four dimensions represented by acronym KSAN (knowledge, skill, attitude and network) mapped on LinkedIn's users profiles. This model is useful to implement our framework to create competent teams with social networks for emergency response. The table 1 shows this notion of competence mapped over the LinkedIn's datasets (Hasse et al. 2017):

Table 1. Mapping the Competence Network with LinkedIn's datasets

\begin{tabular}{ll}
\hline Categories & Datasets LinkedIn \\
\hline Knowledge (K) & Education \\
Skill (S) & Skill \\
Attitude (A) & Volunteer \\
Network (N) & Connections \\
\hline
\end{tabular}

When assembling a team special attention is paid to its structure. The structure itself should be such that the knowledge, skills and character traits (attitude) of team members are complementary. Teams with the same or similar profiles of experts have not proved to be efficient in practice. Besides, practice imposes that, apart from skill and work experience, the criteria for team member selection should include the member character traits and relationships (network). This structure is another relevant data to implement an intelligent system with functionalities for team creation and tasks assignment/delegation. 


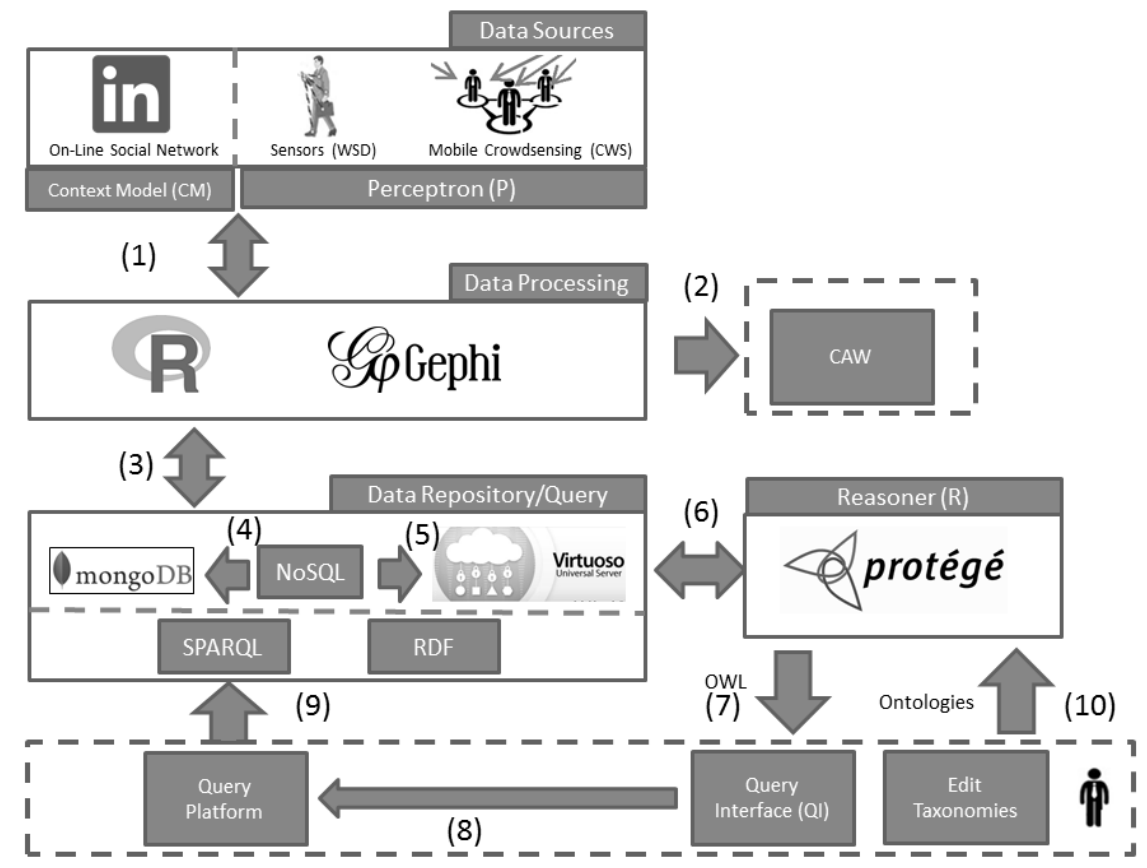

Figure 2. CO-SEMIWA framework

Therefore, the proposed general architecture aims at integrating these three types of platforms: (1) Wearable Sensors Devices (WSD), (2) Crowdsensing System (CWS) and (3) Online-Social Networks (OSNs) to creating an emergency assistance service through an integrated mobile system and this requires the proposition of a new integrative semantic middleware - the Semantic Layer (SL). The Semantic Layer (SL) is formed by our proposed architecture to integrate all these functionalities and platforms in a semantic way. In general, the semantic layer is responsible for all interoperability between systems proposed as components of middleware. We designed these platforms with the same database in a semantic web W3C patterns (OWL 2, RDF, SWRL, RIF). The functionality of CO-SEMIWA seamlessly register all participating devices, aggregates the sensor semantic data, and provides channels to route semantic data to subscribed processes.

Thus, the context model management in emergency scenarios should be considered for ensuring the provision of the right information at the right time and place. As it was pointed in Evchina et al. (2012), ontologies play a crucial role in context-aware systems.

Ontology is a knowledge representation model, which combines the concepts and their relations among each other. Ontologies possess advantages, which make them preferable for use in context-aware systems. They have a set of advantages including human/machine understandable form, capabilities to infer new knowledge, and they are reusable among different domains. On the other hand, ontologies have already been used for discovery services and semantic interoperability among ubiquitous computing devices.

In this research work we integrated context-aware extracting data from WSD, CWS and pre-existing OSNs such as Twitter and LinkedIn, via API, by using extraction and analysis tools like $\mathrm{R}$ and Gephi that provide contextual emergency information. The COLLEGA 
Semantic Middleware (CO-SEMIWA) provides Context-Awareness (CAW) and the functionality to register all devices, acquire the sensor data, unify and aggregate it as semantic data, and route this data to subscribed processes.

The CO-SEMIWA holds all knowledge of the system in a semantic world model. The only requirement for maintaining interoperability with multiple different devices is to provide a common interface for data transmission to the associated process and an application description. Our proposal is to do this using the semantic layer. The CO-SEMIWA framework is shown in Figure 2.

In this paper, we have implementing the CO-SEMIWA semantic layer (SL) like a framework that uses several tools to extract, storage and analyze context of competences from LinkedIn and relevant information of emergencies integrated with emergency data from WSD and the CWS to operate as a single emergency service session in COLLEGA middleware (application layer - AL).

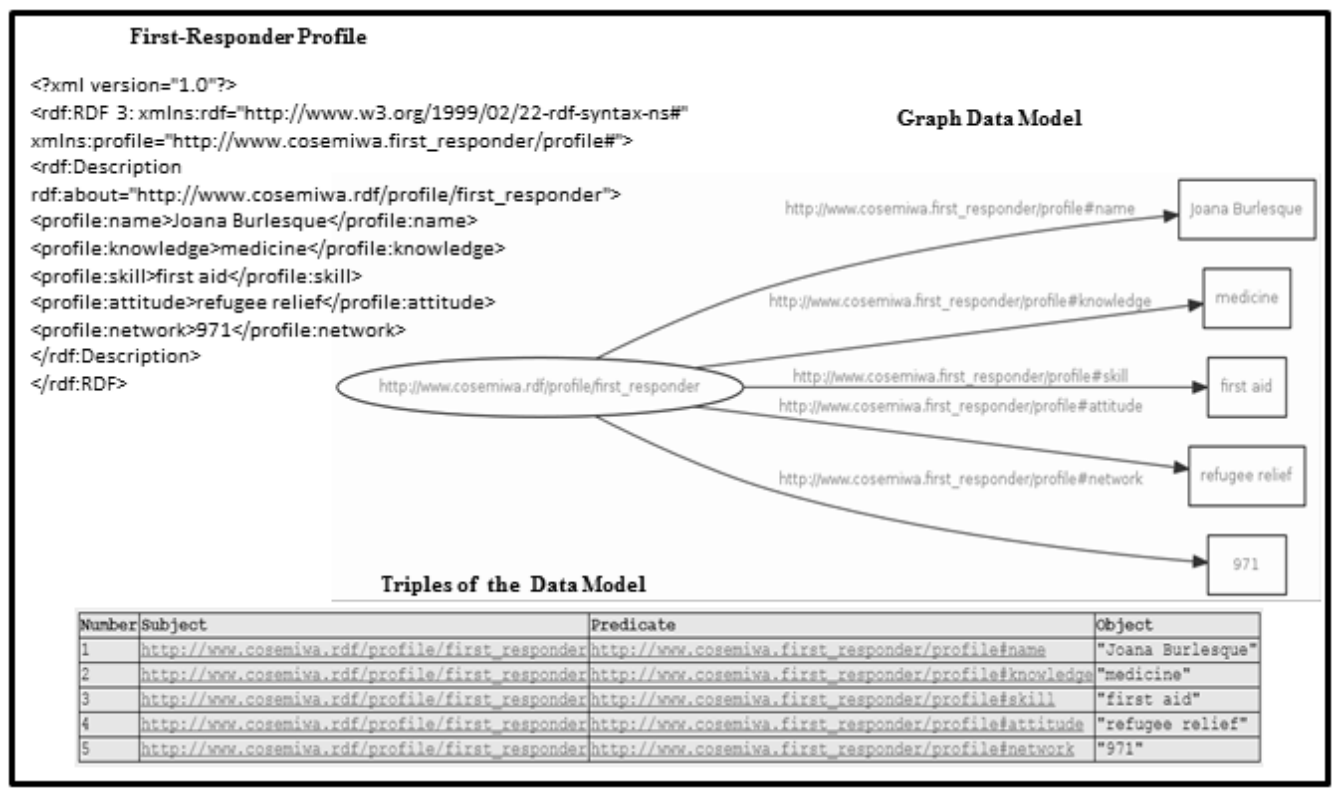

Figure 3. Example of CO-SEMIWA profile

Initially, the Data Sources are obtained from LinkedIn, the WSD and from the COLLEGA Crowdsensing System. They constitute data sources to the components of Semantic Layer Middleware: the Contextual Model (CM) with LinkedIn data and the Perceptron (P) with WSD and crowdsensing data (1).

These data sources are processed by the Data Processing framework that realizes the CAW system module of the CO-SEMIWA architecture (2), by detecting the context and triggering the ECA's module in the COLLEGA middleware (application layer) with the corresponded situation/status flag. Context detection lets ECA's choose (select) the control plan, responding with emergency assistance for the event detected. After detection also are related tasks, profiles and geolocation of the first-responders and victims. 
IADIS International Journal on Computer Science and Information Systems

The Data Processing framework can be implemented using the RStudio platform - which is a complete $\mathrm{R}$ language environment to import and manage data and the Gephi tool. With Gephi tool we can provide all sort of SNA for graph visualization and retrieve metrics to understand the social network relations between users in the system. In this way, by using $\mathrm{R}$ we are developing a computer program that is able to implement the $\mathrm{CM}$ component as follows: 1) extracting competence profiles by LinkedIn's API; and 2) populates NoSQL databases storing data in a RDF format (3). After RDF data process, the NoSQL databases are the central component of the CO-SEMIWA's Framework.

We have deployed one prototype application in $\mathrm{R}$, as shown in the figure 3 that is an example of LinkedIn's user profile (identification data) extraction and transformed in a RDF format (RDF/XML) and respective data model representations to storage the First Responder's profile in the NoSQL databases. The Data Repository/Query manages the processes of data storing and interlinking. The storage contains two different data repositories of NoSQL, RDF triple: MongoDB and Virtuoso databases. NoSQL repository takes charge of maintaining the big datasets that are harvested from various data resources from data on the Web including data generated from the COLLEGA client application in the CWS and WSD. LinkedIn's user information gathered in MongoDB database is constantly analyzed in a batch mode by the Reasoner (R) provided by Protégé environment that is a free, open-source ontology editor and framework for building intelligent systems with a built-in reasoner called HermiT. There are other reasoners available for Protégé, including Pellet and FaCT++ (plug-ins). Basically, new information from all users is searched in MongoDB database through Mongo sub-process (4). User-to-user, with its identification, OWLOnto sub-process (5) performs a search of its existing user profile ontology or a predefined ontological model within Virtuoso NoSQL database of RDF triples. Based on the extracted information, the aim of Interest Emergency and Event Detection sub-process (6) is to generate new emergency event relationships and concepts on the disaster ontology. Over these relationships and concepts, Inference subprocess - OWL (7) deduces new information about interest emergency event and disaster context by the reasoner HermiT in the Protégé to Query Interface (QI).

OntoVirtuoso sub-process (8) allows saving the updated user profile in the Virtuoso NoSQL database with the new information inferred by the reasoner HermiT (Protégé). Once generated or updated the ontological profile for all users, Mongo sub-process set in the MongoDB database that users have been processed. As a result, all relationships generated are stored and can be queried in Virtuoso database, where all sort of information can be accessed without performance problems. In this way, data also can be accessed using a SQL-like query language called SPARQL (9). Finally, to investigate how domain knowledge can help in the acquisition of disasters events we use ontology (by editing taxonomies) (10).

\subsection{Related Work}

The use of mobile software technology to create teams in the domain of disaster and emergency response is still in its infancy. Despite the fact that an emergency situation may develop very dynamically and people should have the right information in a very short time at a mobile location, few projects can be found in the literature developing solutions with all these concerns. 
The Team Phone (Lu et al, 2017) for example, is a system based on smartphones, which provides seamless data communication through several different types of networks and facilitates rescue operations for trapped survivors. The system was designed mainly to network smartphones for providing communications in disaster recovery. By bridging the gaps among different kinds of wireless networks, the system provides smartphones the capabilities of tracking communications since in infrastructures destroyed by disasters. To provide communications in disaster recovery, smartphones, equipped with both cellular and short-range radios (e.g., WiFi, Bluetooth), are the most promising communication tools. In this way, the system acts into the Emergency Response phase, providing functionality for disseminating rescue information during disasters. In emergency response, smartphones have the potential to be the most feasible communication tools. For example, trapped survivors of a structural collapse can communicate with rescue workers and report their position information through the short-range radio (e.g., WiFi) of their smartphones when they are within the communication range of each other. Smartphones of rescue workers can also form networks using WiFi and meet the communication needs in disaster response.

The Argus (Sadhu et al., 2017) is a mobile application for cooperative data collection to build a 3D mapping images of the disaster scene using bystanders present around the incident zone to facilitate rescue operations. The system utilizes this valuable information coming directly from the bystanders and also provides a base framework wherein both the drones and humans can collaboratively work to provide valuable and fine-grained information to the rescue authorities. The Argus system can be used in Emergency Response gathering data (images) from bystanders and Detecting \& Mapping the situation damage in disasters zones.

The First Aid (Surachat et al. 2013) is developed to give some preliminary instructions for taking care of users in Android smartphones. Basically is a navigation system that uses Google API (maps) for searching an appropriate or suitable way or path to the nearest hospital. In the case of any emergency this function is activated on user's smartphone to navigate victims through the shortest path to the hospital. This application provides two types of services, first one is different sets of first aid guidance and second one is finding the appropriate and suitable hospital to go.

The EMuRgency (Kalz et al. 2013) is a cross-border alarm system and a learning programme to teach people how to give cardiopulmonary resuscitation (CPR). The Volunteer-Notification-System (VNS) allows the notification of volunteers nearby a cardiac arrest. An E-Learning Open Educational Resource (OER) is developed on three different levels and in 4 languages allowing institutions to reduce face-to-face training time and to train large numbers in basic CPR skills and knowledge.

The limitation of these approaches is the restrict use to creating and coordinating teams during an emergency situation and managing control plans with a huge number of sensors devices and people involved by the OSNs as the CO-SEMIWA aims to achieve. Another point is that they aren't using an intelligent back-end platform based on ontologies or other artificial intelligence technology. These aspects could in near future limit the range of these solutions in dynamicity and complexity.

In this way, there are some initiatives with the same CO-SEMIWA's approach, like for example in Jing et al. (2014) a mobile context-aware disaster response system called Sky Guard that consists of a mobile client application and a disaster response server.

The Sky Guard Jing et al. (2014) is a context-aware mobile disaster response system which uses mobile client application to capture a variety of user contexts and uses, server modules to analyze these contexts in order to deliver best customized instructions to each user in a proper 
IADIS International Journal on Computer Science and Information Systems

presentation format to the mobile device. It defines what types of the contexts should be included to inform the user activities and disaster environment and explain the process to analyze contexts in details.

The aim of this project is to design a system that provides mobile users with timely and targeted disaster response based on their profile and contexts. In the point of view of Management Phases the Sky Guard system acts in the Emergency Response phase that considers the inherent mobile nature of emergency management and support information exchange operations, gathering data from users and disseminating information using mobile software technologies. This demands a well-designed disaster response system to carefully analyze the contexts of users in emergency to customize and recommend most targeted instructions.

In this regard, recently an interesting trend has emerged in building recommendation systems for disseminating information that leverage the activities of other users on certain things (instructions) to make predictions for the user, by utilizing a methodology, namely Collaborative Filtering.

The collaborative filtering may help to analyze the activities of the users who have similar contexts as a user, and customize the instructions for that user based on the analysis. In a high-level view, the system consists of an ad hoc disaster response team application for disseminating communication which provides all the general disaster response features, a response customization module that uses the collaborative filtering approach and customizes the response that matches with the user's context.

A response presentation module, which determines the presentation format of response information according to the type and features of the user's mobile device, and last, a client application that sits on the mobile device and captures the user's contexts.

The Sky Guard solution is differentiating itself from CO-SEMIWA by not adopting semantic web technology in the project. On the other hand, in Cordeiro et al. (2011) the proposed system is using the Linked Data's approach in the design of information infrastructure for Collaborative Emergency Management System extensively adopting the semantic Web technology and creating teams to act in a disastrous scenario, but this solution doesn't have been focused for mobile applications and crowdsensing technology, or using OSNs to amplify the scope of users scalability.

\section{CONCLUSION}

In this paper we identified and investigated the main issues related to handle with creating competent teams for emergency response using data collected by social networks and emergent technologies required such as Crowdsensing Systems, Wearable Sensors Devices, algorithms and intelligent systems (e.g. Ontologies, SNA).

As a result we propose a novel framework to integrate all requirements to provide a platform with capabilities to manage all life cycle of emergency assistance efforts for the response phase in the emergency management.

For this, we presented the CO-SEMIWA architecture/framework, a semantic middleware for collaborative assistance in emergency scenarios. Semantic information is provided using semantic web patterns by addressing various Wearable sensors devices for users' interaction, 
through a Crowdsensing System and required competences extracted from OSNs such as LinkedIn to create competent teams.

For future work this platform will be fully operational for conduct a practical use case with semantic data for testing and creating competent teams in a research environment of the emergency response area.

\section{ACKNOWLEDGEMENT}

This research was supported by the program CAPES $3^{\circ}$ Cronograma - Chamadas de Projetos $\mathrm{n}^{\circ}$ 09/2014, processo $\mathrm{n}^{\circ}$ 8745-13-7 for Carlos Roberto De Rolt and $\mathrm{n}^{\mathrm{o}}$ 88887.122829/2016-00 for Danilo Hasse.

\section{REFERENCES}

Abu-Elkheir, M. et al, 2016. Enhancing Emergency Response Systems through Leveraging Crowdsensing and Heterogeneous Data Analytics. Proceedings 2016 International Wireless Communications and Mobile Computing Conference (IWCMC). Paphos. Cyprus, pp. 188-193.

Anagnostopoulos A. et al, 2012. Online team formation in social networks. Proceedings of the 21st international conference on world wide web, $W W W^{\prime} 12$. New York, USA, pp 839-848.

Araujo, F.C.S. and Borges, M.R.S., 2012. Support for Systems Development in Mobile Devices used in Emergency Management. Proceedings of the 2012 IEEE 16th International Conference on Computer Supported Cooperative Work in Design. Wuhan, China, pp. 200-206.

Awal, G.K. and Bharadwaj, K.K., 2014. Team formation in social networks based on collective intelligence - an evolutionary approach. In: International Journal of Research on Intelligent Systems for Real Life Complex Problems, Vol.41, No. 1, pp.627-648.

Bessai, K. and Charoy F., 2016. Business Process Tasks-assignment and Resource Allocation in Crowdsourcing Context. Procceedings 2nd International Conference on Collaboration and Internet Computing. Pittsburgh, USA, pp. 11-18.

Bonchi F. et al, 2011. Social network analysis and mining for business applications. In: ACM Trans Intell Syst Technol, Vol.2, No.3, pp.22.1-22.37.

Carter, L. et al, 2014. Social Media and Emergency Management: Exploring State and Local Tweets. Proceedings 47th Hawaii International Conference on System Science (HICSS. Waikoloa, USA, pp. 1968-1977.

Cong L. et al, 2015. E-Net Modeling and Analysis of Emergency Response Processes Constrained by Resources and Uncertain Durations. In IEEE Journals \& Magazines: Transactions on Systems, Man, and Cybernetic, Vol. 45, No. 1, pp. 84-96.

Cordeiro, K.F. et al., 2011. Use of Linked Data in the Design of Information Infrastructure for Collaborative Emergency Management System. Proceedings of the 2011 15th International Conference on Computer Supported Cooperative Work in Design (CSCWD). Lausanne, Switzerland, pp.764-771.

Corradi, A. et al, 2015. Automatic Extraction of POIs in Smart Cities: Big Data Processing in ParticipAct. Proceedings IFIP/IEEE International Symposium on Integrated Network Management (IM2015): Experience Session Paper. Ottawa, Canada, pp. 1059-1064.

Cova, T., 1999. GIS in Emergency Management. In book: Geographical Information Systems: Principles, Techniques, Applications, and Management. John Wiley \& Sons, New York, USA. 
IADIS International Journal on Computer Science and Information Systems

Crowe, A., 2010. The social media manifesto: A comprehensive review of the impact of social media on emergency management. In Journal of Business Continuity \& Emergency Planning. Vol 1, No. 5, pp. 409-420.

De Rolt, C.R. et al, 2017. Competence-based mobile Community Response Networks. Proceedings IEEE Symposium on Computers and Communications (ISCC). Heraklion, Greece, pp.994-1000.

Evchina, Y. et al, 2012. Ontological Framework of Context-Aware and Reasoning Middleware for Smart Homes with Health and Social Services. Proceedings IEEE International Conference on Systems, Man, and Cybernetics. Seoul, Korea, pp.985-990.

Gale, D., 2001. The two-sided matching problem. Origin, development and current issues. In: International Game Theory Review, Vol. 3, Nos. 2 \& 3, pp. 237-252.

Gajewar A. and Sarma A.D., 2012. Multi-skill collaborative teams based on densest subgraphs. Proceedings of the 12th SIAM international conference on data mining, SDM'12, Anaheim. California, USA, pp. 165-176.

Geumpana, T.A. et al, 2015. Mobile Cloud Computing for Disaster Emergency Operation: A Systematic Review. Proceedings IEEE International Symposium on Technology in Society (ISTAS). Dublin, Ireland, pp. 1-8.

Gu, T-J. et al, 2017. Developing an Emergency Response Conceptual Framework for Network Centric Disaster Operations. Proceedings IEEE 3rd International Conference on Information Management. Chengdu, China, pp. 252-257.

Haghighi, P.D. et al, 2010. Ontology-Based Service-Oriented Architecture for Emergency Management in Mass Gatherings. Proceedings IEEE International Conference on Service-Oriented Computing and Applications (SOCA). Perth, WA, Australia, pp.1-7.

Harb, M. and Dell'acqua, F., 2017. Remote Sensing in Multirisk Assessment: Improving disaster preparedness, in IEEE Geoscience and remote sensing magazine, Vol.5, No.1,pp 53-65.

Hasse, D. et al, 2017. Formação de Redes de Competências a partir dos Perfis em Redes Sociais: Um Experimento com Datasets do Linkedin. Proceedings Iadis Conferências Ibero Americanas WWW/Internet. Algarve, Portugal, pp.12-20.

Jaimes, L.G et al., 2015. A Survey of Incentive Techniques for Mobile Crowd Sensing. IN IEEE Internet of Things Journal, Vol. 2, No.5, pp. 370-380.

Jing, N. et al., 2014. A Context-aware Disaster Response System Using Mobile Software Technologies and Collaborative Filtering Approach. Proceedings of the 2014 IEEE 18th International Conference on Computer Supported Cooperative Work in Design. Hsinchu, Taiwan, pp.516-522.

Kalz, M. et al, 2013. EMuRgency: Addressing cardiac arrest with socio-technical innovation in a smart learning region. In Interaction Design and Architectures Journal. Vol 17(2), pp.77-91.

Lappas T., et al., 2009. Finding a team of experts in social networks. Proceedings of the 15th ACM SIGKDD International Conference on Knowledge Discovery and Data Mining, KDD '09. New York, USA, pp. 467-476.

Luqman F. and Griss M., 2010. Overseer: A Mobile Context-Aware Collaboration and Task Management System for Disaster Response, Proceedings Eighth International Conference on Creating, Connecting and Collaborating through Computing. La Jolla, USA, pp.76-82.

Li C.T. and Shan M.K., 2010. Team formation for generalized tasks in expertise social networks. Proceedings of the IEEE 2nd international conference on social computing SOCIALCOM '10. Washington, DC, USA, pp. 9-16.

Li, Yan, 2011. A Survey on Communication Networks in Emergency Warning Systems. Report Number: WUCSE-2011-100 (2011). All Computer Science and Engineering Research.

Li, T., et al, 2017. Data-Driven Techniques in Disaster Information Management, In ACM Computing Surveys (CSUR), Vol. 50, No. 1, April. 


\section{COORDINATING EMERGENCY RESPONSE BY COMPETENT TEAMS}

Liu, C. et al, 2015. E-Net Modeling and Analysis of Emergency Response Processes Constrained by Resources and Uncertain Durations. In IEEE Transactions On Systems, Man, and Cybernetics Systems, Vol. 45, No. 1, pp.84-96.

Lu, Z. et al, 2017. TeamPhone: Networking Smartphones for Disaster Recovery. In IEEE Transactions on Mobile Computing, Vol. 16, No.12, pp. 3554-3567.

Luna, S. and Pennock, M., 2015. Social Media in Emergency Management advances, challenges and future directions. Proceedings Annual IEEE Systems Conference (SysCon) Proceedings. Vancouver, BC, Canada, pp. 792-797.

Mehrotra, S. et al, 2013. Technological Challenges in Emergency Response. In Guest Editors' Introduction, IEEE Computer Society, Vol.28, No.4, pp.5-8.

Moi, M. et al, 2016. An Ontology for the Use of Quality Evaluated Social Media Data in Emergencies. In IADIS International Journal on WWW/Internet, Vol. 14, No. 2, pp. 38-57.

Moore, T., 2008. Disaster and Emergency Management Systems. ISBN 978-0-580-60710-3, British Standards Institution, UK.

Murphy, R.R., 2016. Emergency Informatics: Using Computing to Improve Disaster Management. In Computer, Vol. 49, No. 5, pp. 19-27.

Nazer,T.H., 2017. Intelligent Disaster Response via Social Media Analysis A Survey. In ACM SIGKDD Explorations, Vol. 19, No. 1.

Palen, L. and Liu, S., 2007. Citizen Communications in Crisis: Anticipating a Future of ICT Supported Participation. Proceedings of the SIGCHI Conference on Human Factors in Computing Systems. San Jose, USA, pp. 727-736.

Pop, B. and Boian, F., 2014. Algorithms for Automating Task Delegation in Project Management, Proceedings of the 2014 Federated Conference on Computer Science and Information Systems. Warsaw, Poland, pp. 1191-1196.

Pavković, B., Milenković, M., 2014. Situation awareness and decision support tools for response phase of emergency management: a short survey. Proceedings IEEE 25th International Workshop on Database and Expert Systems Applications. Munich, Germany, pp. 154-159.

Rosas E. et al, 2016. Survey on Simulation for Mobile Ad-Hoc Communication for Disaster Scenarios. In Journal of Computer Science and Technology, Vol.31, No.2, pp. 326-349.

Sadhu, V. et al., 2017. Argus: Smartphone-enabled Human Cooperation for Disaster Situational Awareness via MARL. Proceedings IEEE International Conference on Pervasive Computing and Communications Demonstrations. Kona, HI, USA, pp.79-81.

Surachat, K. et al, 2013. First Aid Application on Mobile Device. In International Scholarly and Scientific Research \& Innovation. Vol.7(5), pp-361-366.

UNISDR, 2009. UNISDR Terminology On Disaster Risk Reduction. United Nations International Strategy for Disaster Reduction. Geneva, Switzerland.

Vivacqua, A.S. and Borges, M.R.S., 2001. Taking advantage of collective knowledge in emergency response systems. in Journal of Network and Computer Applications,

Weaver, A.C. et al, 2016. Computing Tools and Techniques for Emergency Response. In IEEE Computer Society, Vol.49, No.5, pp. 16-18.

Whittaker, J. et al., 2015. A review of informal volunteerism in emergencies and disasters: Definition, opportunities and challenges. International In Journal of Disaster Risk Reduction, Elsevier.

Wi H., et al., 2009. A Team Formation Model Based on Knowledge and Collaboration. In Journal Expert Systems with Applications. Vol. 36, No.5, pp. 9121-9134. 